\title{
The Design of Variable Stiffness Massage head Based on Magnetorheological Fluid
}

\author{
ZhiCheng Xu, Xing Ming, YiKai Shi", and WenDong Wang \\ School of Mechanical Engineering, Northwestern Polytechnical University, China
}

\begin{abstract}
A controlled massage head is designed to vary the massage intensity rapidly. It bases on the property of magnetorheological fluid stiffness influenced by magnetic field. In this paper, we draw on relevant methods, design electromagnetic structures with numerical analysis software. The method of positive and negative current with permanent magnet compensation is presented to achieve a bigger range of magnetic field strength adjustment. Further, the massage head prototype is manufactured and tested. The experiment data are processed and indicates the design of the magnetic field strength and massage strength can meet the requirements.
\end{abstract}

Keywords magnetorheological fluid; massage instrument; electromagnetic simulation; structure design

\section{Introduction}

Massage has always been one of the effective means of relieving and treating chronic pain, approved and trusted by lots of people [1]. Traditional massage needs to be done by experienced masseurs, not only with high costs, but also the number of the masseurs is not enough comparing with public requirements. To solve this problem, many companies have produced a variety of massage machines on the market, such as massage hammer, massage chair, massage pillow, etc. However, these massage machine structure and functions are too simple with poor flexibility. They are unable to adjust the massage intensity in time for different massage situation also can not satisfy the personalized requirements. In view of the above disadvantages, we present a variable stiffness massage head based on magnetorheological fluid. MRF is a new controllable fluid material, its property can change within a few milliseconds from Newtonian fluid to structure which is similar to solid and controlled by magnetic field [2].At present, the magnetorheological fluid is mainly used in the design of semi-active shock absorbers and dampers, and some mature products have been come out [3]. Apart from this, there are many researchers committed to the application of controllable stiffness and deformation property of magnetorheological fluid. Yuki OKATANI from Kyushu Institute of Technology designed a MR fluid robot gripper, it is able to grab different shapes of objects weight $5 \mathrm{~kg}$ approximately [4]; Jin Xing Kong put MRF into thin spherical shell enhancing its stiffness to improve process precision [5] Zhang XZ from University of Science and Technology of China analyzed the flexible fixture based on MRF, and carried out theoretical and experimental studies on the extrusion enhancement effect of MRF [6]. According to these features such as fast response to magnetic field, the stable state transition process and the controllable stiffness, the design idea is presented of using the magnetorheological fluid in semi-active control massage head.

\section{The massage head electromagnetic structure design and methods}

According to the theory of electromagnetism, the attenuation of the magnetic field is proportional to the square of the distance. Therefore, the circular massage head ensures the most efficient use of the magnetic field generated by the electromagnetic coil. Disposed outside of the coil is the yoke connect to the iron core, in order to reduce the air gap of the magnetic circuit and also suppress the magnetic flux leakage. The material of massage head surface is silicone. Silicone is non-toxic and odorless, the chemical properties are stable and most important it's insoluble in MRF base fluid [4]. We use MRF-140CG of LORD Company for massage head working stuff. The yield strength and B-H curve of the MRF are shown in figure 1 [7], the massage intensity designed for $0 \sim 30 \mathrm{~N}$. The yield strength of the MRF is designed about $10 \mathrm{kPa}$. According to the data in Fig. 1, the magnetic field strength is initially designed to be $20 \mathrm{kA} / \mathrm{m}$. The MRF massage head structure schematic diagram is shown in figure 2 . The coil connected to DC generat es a controlled magnetic field, and the massage head MRF influenced by the magnetic changes the characteristic. Adjust the current in the coil, affect and change the yield strength of the magnetorheological fluid, thus alter the contact stiffness with human, finally control the massage intensity.

\footnotetext{
*Corresponding author: ykshi@nwpu.edu.cn
} 


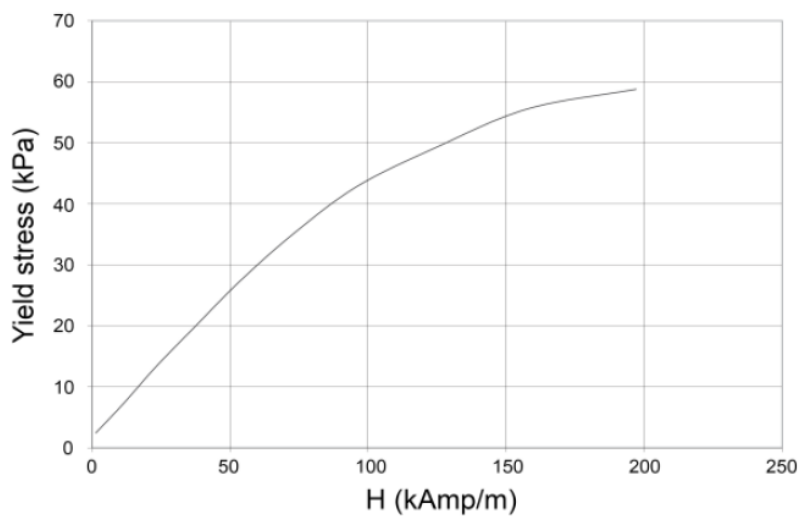

(a)

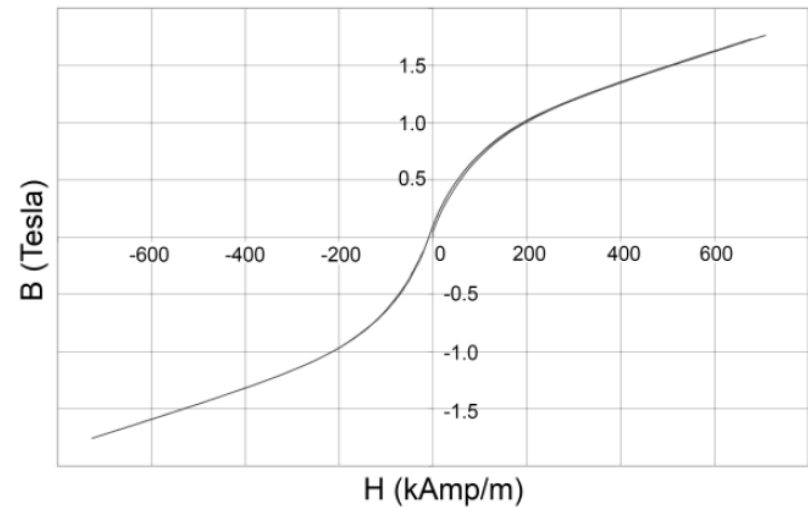

(b)

Fig. 1. MRF-140-CG Yield stress - field correlation (a) and BH curve (b)

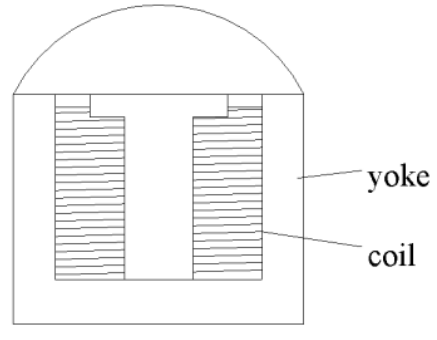

Fig. 2. The structure schematic diagram

The theoretical basis for the analysis of electromagnetic is the Maxwell Equations, as for the structure designed, ignore the propagation of electromagnetic waves, the current and the field change slowly with time, it meets the special case of Maxwell's equations:

$$
\begin{gathered}
\nabla \times \vec{E}=-\frac{\partial \vec{B}}{\partial t} \\
\nabla \times \vec{H}=\vec{J} \\
\nabla \vec{D}=0 \\
\nabla \vec{B}=0 \\
\nabla \vec{J}=0
\end{gathered}
$$

Here $E$ is the electric field intensity, $B$ is the magnetic flux density, $H$ is the magnetic field strength, $J$ is the current density, $D$ is the power density. Also:

$$
\vec{D}=\varepsilon \vec{E} ; \vec{B}=\mu \vec{H} ; \vec{J}=\sigma \vec{E}
$$

$\varepsilon$ is the dielectric constant of the material, $\mu$ is the permeability of the material, and $\sigma$ is the conductivity of the material.

However, even with the boundary conditions, it is very difficult to solve the above equations. The traditional electromagnetic field calculation method is usually used mathematical and physical simulation method or approximate analysis method. These methods are not accurate, often need to be compensated according to the measured values after the completion of the design to meet the requirements of the field distribution [8]. Magnetic circuit method is also widely used to calculate magnetic field, it has the advantage of simplicity and the result is accurate when the air gap is small. But it can't be used for the open magnetic field calculation. Therefore, we refer to the empirical formula of electromagnet design [8]:

$$
N I \approx \frac{B L_{\text {air }}}{4 \pi \times 10^{-7}}
$$

Here $B$ is the magnetic induction of the target point, and $L_{\text {air }}$ is the working air gap of the target point.

The preliminary calculation indicates a large magnetic flux about 3500Amp, But the size of massage head shouldn't be too large, we design using wire diameter $0.75 \mathrm{~mm}$, rated current $2 \mathrm{~A}, 1500$ turns of the DC coil, and add some permanent magnet as the magnetic field compensation to reach the demand of magnetic field. The compensation details are described in section 2.2.

\section{Simulation and optimization}

\subsection{Ansoft Maxwell simulation}

With the development of computer technology, the numerical analysis method of electromagnetic field calculation is widely used, including the finite difference method, the finite element method and the boundary element method. Based on these methods, some computer programs and software are designed for calculation, we can get the magnetic field distribution accurate results simply and fast. Ansoft Maxwell finite element numerical analysis software is an effective way to solve the problem of electromagnetism, it is widely used in the lowfrequency electromagnetic field analysis. We use it to revise the preliminary results by finite element analysis and get the effective value. Maxwell's intuitive and convenient modeling interface and the abundant material database make structure model building efficiently. Establish a massage head mechanical structure model and select the material for different parts. According to the coil size, design the size and material of the yoke and the iron core: The core through the coil frame, the length is larger than the yoke height, choose DT4 electric iron because of 
its high permeability and coercivity with big magnetic saturation; Yoke slightly larger than the coil in diameter, $4 \mathrm{~mm}$ thick, higher than the coil height of about $5 \mathrm{~mm}$ for the placement of wire and the coil cover, the material choose No.10 steel for big magnetic saturation. There is no data of DT4 and MRF in Maxwell material library, we can add them to the project material library conveniently according to the B-H curve in figure 1 and the DT4 data in reference [8].The data and progress are shown in figure 3.

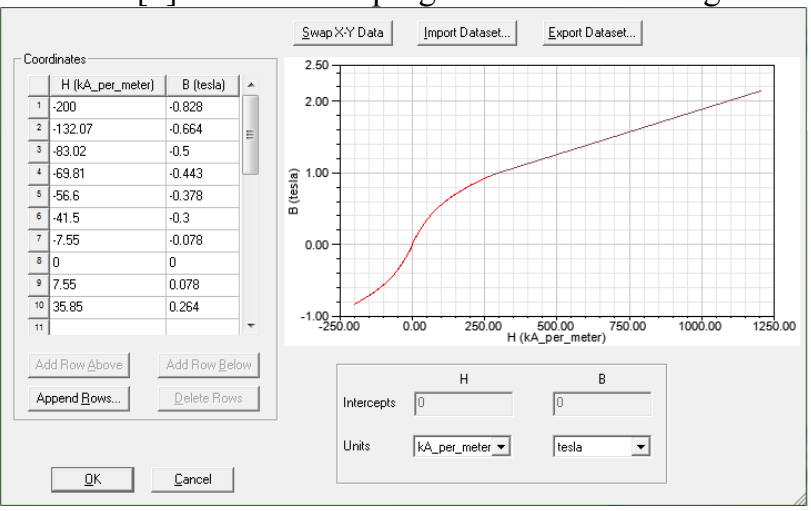

(a) The B-H curve of MRF

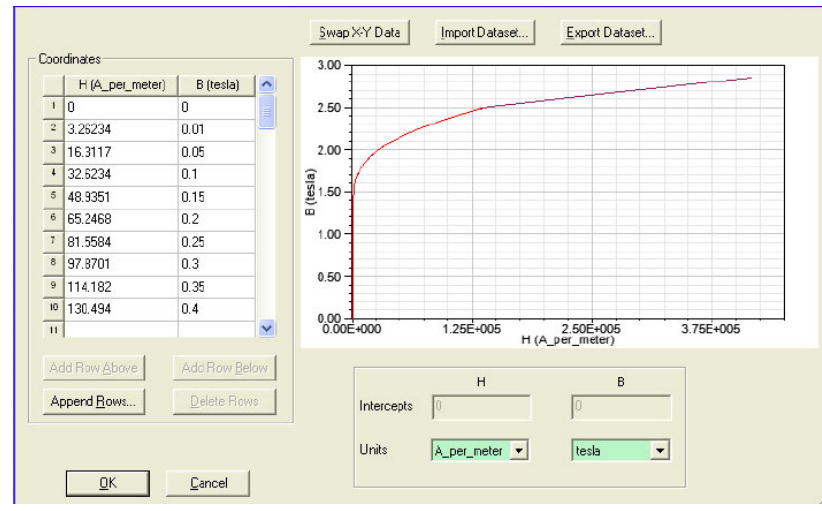

(b) The B-H curve of DT4

Fig. 3. Add property curve of DT4 and the MRF to Maxwell

\section{2 magnetic field compensation}

According to the information in Figure 1, the results of calculation and simulation (Fig.4) indicate that the magnetic field generated by the coil is not sufficient to make the magnetorheological fluid achieve the desired yield strength for the massage. Therefore, on the basis of the magnetic field superposition principle, it is necessary to place additional permanent magnets outside the core coil to ensure the magnetic field strength. We found that with the increase of the working point axial distance, the attenuation of magnetic field intensity is the main cause of the deficiency of the magnetic field. Common permanent magnets are disc-shaped, but it can't decrease the axial distance efficiently. Hemispherical permanent magnets are better for magnetic field compensation, however, the manufacture of hemispherical magnet is difficult, magnetizing is hard to be uniform, and the magnetic energy product is worse than the chip magnet. In order to ensure the effective massage volume of the massage head, we design with round-shaped $\mathrm{NdFeB}$ magnets as compensation, the simulation results are as follows.

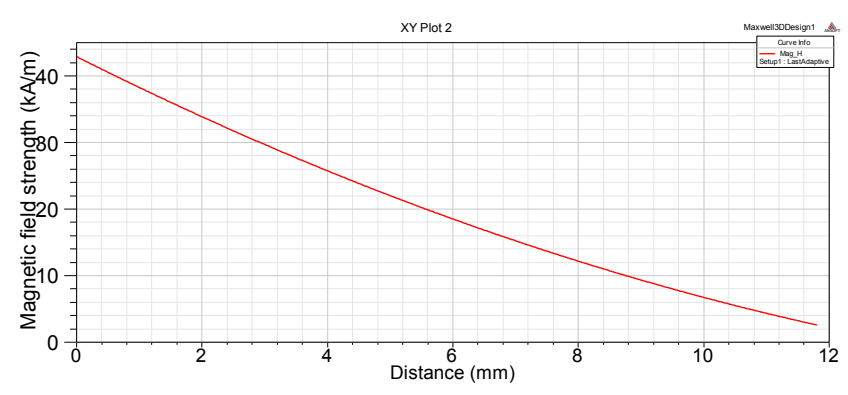

Fig. 4. Axial magnetic field simulation result without magnets

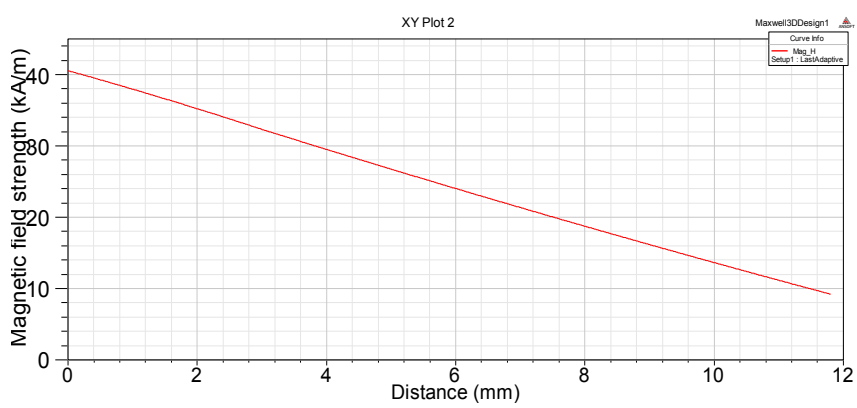

Fig. 5. Axial magnetic field simulation result with magnets

After the addition of permanent magnets, the magnetic field still maintains a high level when the current in the coil is very small. This is contrary to the original intention of our design, the stiffness of MRF cannot be well controlled. So we propose that reverse the current in the coil to offset some part of the magnets magnetic field in order to the bigger range of changes in the stiffness of the massage head. The intrinsic coercive force $H_{c j}$ is a physical quantity that measures the anti-demagnetization ability of the magnet. It means the coercivity when the magnetization in the material is zero. It is an important index to judge whether the permanent magnet material can maintain its original magnetization. When the current reversed, the magnetic field strength generated by the coil is $129.3 \mathrm{kA} / \mathrm{m}$, calculated by Maxwell. It is far less than the intrinsic coercivity of the $\mathrm{NdFeB}$ magnets, so that a reasonable superposition of the magnetic field can be achieved. The MRF working magnetic field strength when the current is negative or positive are shown in Figure 6.

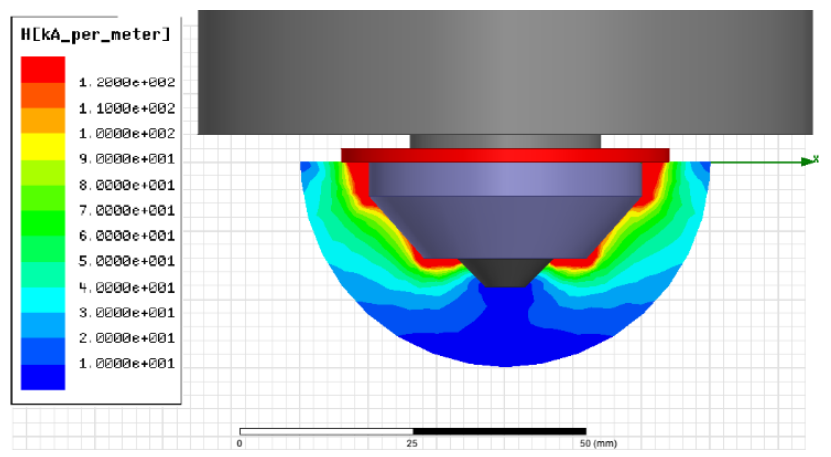

(a) The magnetic field strength in negative current 


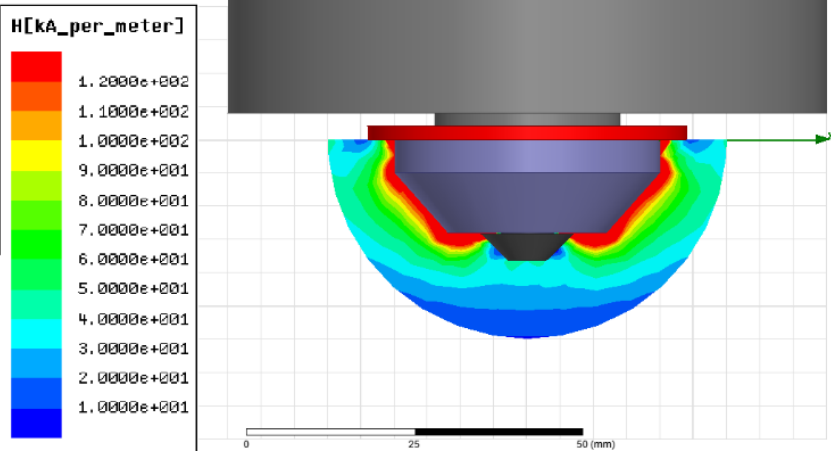

(b) The magnetic field strength in positive current

Fig. 6. The magnetic field strength situations

\subsection{Sealed structure design and mechanical construction}

Magnetorheological fluid is an oily liquid based material, it has fluidity without the influence of magnetic field. Leakage of the MRF may damage the power coil, also contaminate massage head surface affecting the experience of users. Therefore, the seal of the MRF is also an important part of the mechanical structure design. We design to seal the MRF in a hemispherical silicone massage head. The massage head edge apply squeeze-type seal, use the shell with a trench thinner than the silicone membrane to fix. The shell is bolted to connect the coil cover and the yoke to seal the edge of the silicone membrane. The $\mathrm{NdFeB}$ magnets have center throughholes, put a sealing ring into the notch of the coil cover on the large side of magnets, meanwhile the sealing ring separates the iron core from the magnets to reduce the influence of the magnetic saturation; Set a special low carbon steel bolt through the magnet center through-hole and the gasket over the small side of the magnet, tight them with the iron corn to seal the MRF. The final design massage head structure is shown in Fig.7

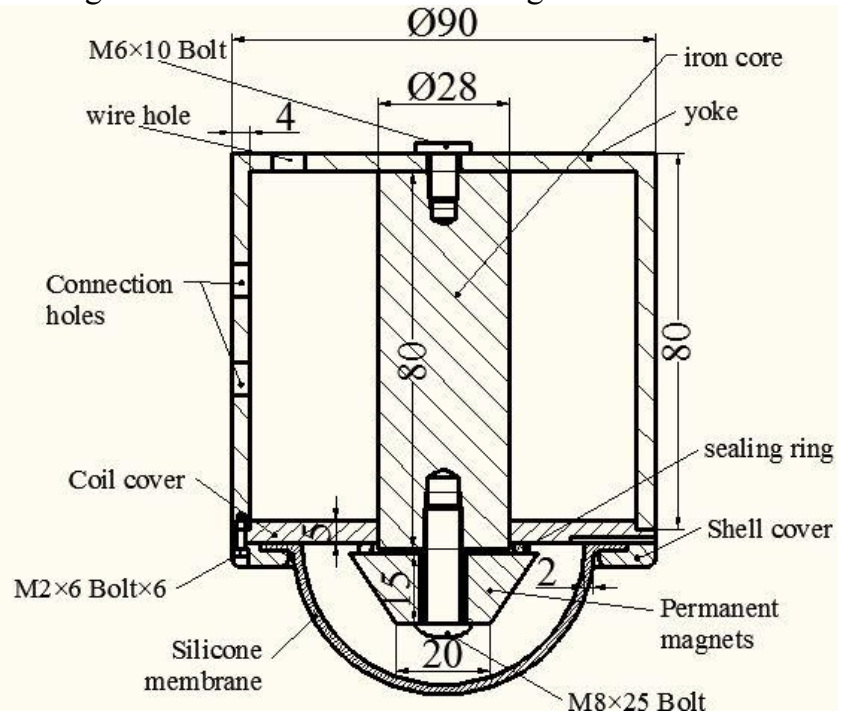

Fig. 7. The Massage Head structure

\section{Experiment and data process}

\section{1 measure of the magnetic field}

The stiffness of MRF is affected by the magnetic field, so whether the massage force can reach the expected aim or not depends on the magnetic field. To prove the simulation results are correct, we use a gauss meter to measure the magnetic field after the structure is designed and assembly. The gauss meter cannot be used in MRF, so we have to measure the field in the air. Therefore, the air is the magnetic medium in simulation, set "None model" for the MRF part in Maxwell, don't change any others parameter of the magnetic only the medium. If the simulation results match the measured data, it can be considered that the simulation results are correct in the MRF medium. We found there is significant disparity of the data in different times of experiment due to the manual measure and the high sensitivity of the gauss meter in milliTesla degree. Therefore, we use MATLAB to deal with it. The data are shown in table 1.

Table 1. The measure data of magnetic flux density in different distance

\begin{tabular}{|c|c|c|c|c|c|}
\hline $\begin{array}{l}\text { Distance from the } \\
\text { magnet } / \mathrm{mm}\end{array}$ & 1 & 3 & 5 & 7 & 9 \\
\hline \multirow{4}{*}{$\begin{array}{l}\text { The magnetic flux } \\
\text { density of measure } \\
\text { points in positive } \\
\text { current (mT) }\end{array}$} & 254.3 & 193.8 & 153.8 & 126.9 & 104.9 \\
\hline & 270.7 & 192.2 & 151.7 & 125.1 & 107.5 \\
\hline & 262.0 & 199.2 & 157.6 & 129.1 & 104.7 \\
\hline & 269.3 & 202.0 & 154.0 & 126.8 & 106.6 \\
\hline \multirow{4}{*}{$\begin{array}{l}\text { The magnetic flux } \\
\text { density of measure } \\
\text { points in negative } \\
\text { current (mT) }\end{array}$} & 104.1 & 89.0 & 74.3 & 55.3 & 44.7 \\
\hline & 114.3 & 90.3 & 69.8 & 54.4 & 42.3 \\
\hline & 112.4 & 83.8 & 67.2 & 53.9 & 42.7 \\
\hline & 105.8 & 85.0 & 70.2 & 53.0 & 42.8 \\
\hline
\end{tabular}

Matlab curve fitting tool has powerful curve fitting ability and provides various type of objective functions. The magnetic field of massage head is made up of 2 parts and the magnetic flux density decreases till 0 with the distance increases. Comparing different fitting curves we find that the exponential double terms function approximates the physical principles of magnetic and its $\mathrm{R}$-square is $99.58 \%$, so we choose this as our objective function. The compare between the fitting curve and Maxwell simulation curve are shown in Fig. 8

It indicates that the measured data basically meet the magnetic flux density required for the yield strength of $\mathrm{MRF}$, and the positive and negative current can make the magnetic field strength in a relatively large range of control. However, the simulation results and experimental measurements have a great diversity. As for the reason, maybe the special low-carbon steel bolt material is not standard, the magnetic field through its conduction is limited; or maybe the seal ring and the gasket are squeezed to cause the working distance from the magnet smaller compared with the simulation, which led to a deviation of the curve. Overall, the electromagnetic structure we 
designed effectively controlled the massage head magnetic field

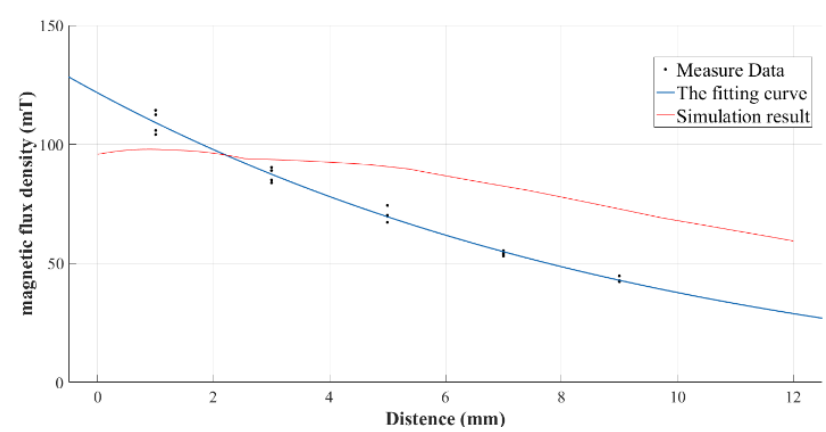

(a) The fitting curve of data in positive current

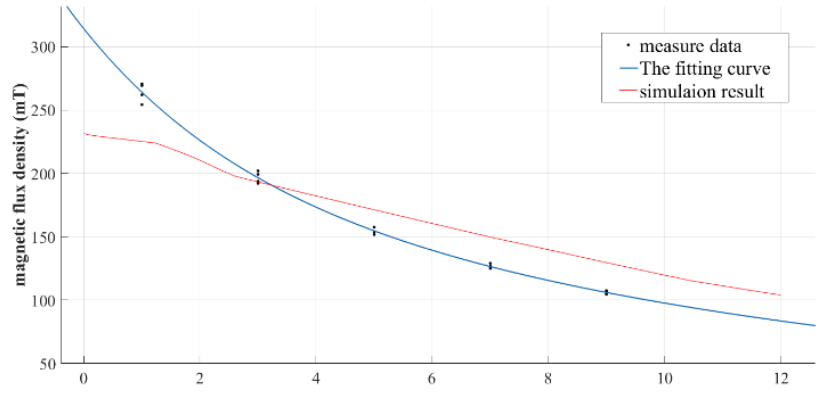

(b) The fitting curve of data in positive current

Fig. 8. The curve comparison between experiment and simulation

\section{2 massage force measure}

After filling and sealing the MRF, use the sensor to measure the massage intensity at different current. When the MRF head is observed to be obviously out of shape by pressure, record the sensor data. The data are shown in table 2 .

Table 2. The massage head yield force in different current.

\begin{tabular}{|l|l|l|l|l|l|l|l|l|l|}
\hline Current/A & -2 & -1.5 & -1 & -0.5 & 0 & 0.5 & 1 & 1.5 & 2 \\
\hline Yield force/N & 2.4 & 4.5 & 5.9 & 7.5 & 10.6 & 14.8 & 19.9 & 22.1 & 25.5 \\
\hline
\end{tabular}

The result of the experiment shows that the massage head fulfills the demand of different massage force with fast response.

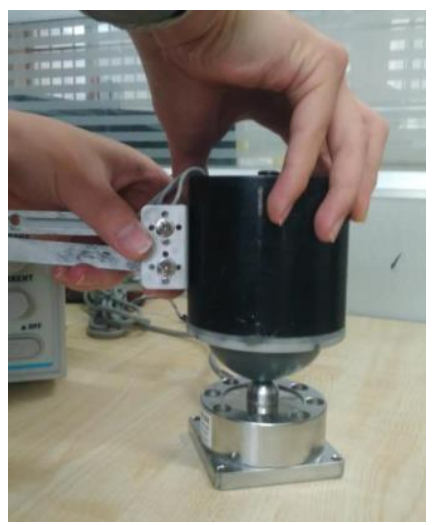

Fig. 9. The experiment of massage head yield force test

\section{Conclusion.}

We design a controllable massage head based on the MRF. First we analyzed the massage head requirements, and demonstrated the feasibility of variable stiffness. Next, design the electromagnetic structure of the massage head according to the prospective massage force and simulate in computer software. Moreover, design the permanent magnet compensation for the insufficient magnetic field drawing on the classic electromagnet design method, and present the idea of forward and reverse excitation current for better control. Then, design the structure and seal of the massage head, manufacture the prototype and test, deal with the experimental data by mathematical process. The results show that the variable stiffness massage head can effectively achieve the transformation of different massage intensity to meet the design requirements.

In future studies, the miniaturization of the massage head, the more freedom degrees of the massage machine, the optimization design of the magnetic field will be improved.

\section{References}

1. Huang, Y., Li, J., Huang, Q., \& Souères, P. (2015). Anthropomorphic robotic arm with integrated elastic joints for TCM remedial massage. Robotica, 33(02), 348-365.

2. X. Zhang, F. Ma, F. Zhao, K. Guo, and M. Ahmadian, "Optimization of the Magnetic Property of a Magnetorheological Squeeze Mount," The FISITA 2012 World Automotive Congress, Beijing, (2012), p. 14.

3. F. Imaduddin, S. A. Mazlan, and H. Zamzuri, "A design and modelling review of rotary magnetorheological damper," Materials \& Design, vol. 51, pp. 575-591, (Oct 2013).

4. Y. Okatani, T. Nishida, K. Tadakuma, and Ieee, "Development of Universal Robot Gripper Using MR alpha Fluid," $7^{\text {th }}$ Scis and $15^{\text {th }}$ Isis, pp. 231-235, (2014).

5. J. X. Kong, Y. C. Zheng, and X. K. Wang, "Fixture Design Based on Magneto-rheological Fluids for Thin Wall Spherical Shell Precision Machining," in Digital Design and Manufacturing Technology Ii. vol. 215, C. Lu, Ed., ed, (2011), pp. 315-319.

6. X. Z. Zhang, Q. M. Wang, P. Q. Zhang. "The flexible fixture based on Magnetorheological Fluids," Journal of Experimental Mechanics, No.2 vol. 18, pp. 185-192, (2003).

7. A. Spaggiari and E. Dragoni, "Effect of Pressure on the Flow Properties of Magnetorheological Fluids," Journal of Fluids Engineering-Transactions of the Asme, vol. 134, (Sep 2012).

8. Q. F. Li, Numerical Calculation of Electromagnetic Fields and Design of Electromagnets. (TsingHua University, 2002). 